https://www.journal-imab-bg.org
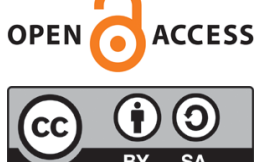

\title{
THREE DIMENSIONAL VIRTUAL PLANNING, MODELLING AND PRINTING IN FIBULA FREE FLAP MANDIBULAR RECONSTRUCTIONS - INI- TIAL CASE SERIES IN A LIMITED RESOURCE ENVIRONMENT
}

\author{
Nikolay Yanev, Svetoslav Slavkov, Daniel Markov, Alexandrina Malinova, \\ Maxillofacial Unit, University Multiprofile Acute Hospital "N. I. Pirogov", So- \\ fia, Bulgaria.
}

\begin{abstract}
The most contemporary approach when dealing with extensive head and neck defects post oncological resections is based on virtual computer planning, surgical resection/ reconstruction simulation and three dimensional modeling and printing. It is of great benefit, particularly when the maxillo-mandibular area is involved, because of its complex three dimensional form and active functional load, both these features presenting a long-standing reconstructive challenge to the head and neck surgeons.

Developing such complex surgical techniques is a very resource dependent undertaking and relies substantially on a strategic support from the given National Health Service system.

An overview of the first 3 consecutive cases of entirely digitally planned and lab-to-theatre transferred mandibular resections and simultaneous microvascular fibula flap reconstructions performed in Sofia between August October 2017 is presented.
\end{abstract}

Keywords: Computer-Assisted Therapy, Planning Technic, Fibula, Microsurgical Free Flaps, Mandibular Reconstructive Surgery,

\section{INTRODUCTION}

The oncologic head and neck surgery often leads to various tissue defects. The latter predisposes a major functional and aesthetic impairment, reduced quality of life and social isolation of patients.

The reconstruction of large defects resulting from surgical treatment is a challenging and demanding problem.

The mandible has an important role in maintaining the alimentary function, respiratory function and speech and is also responsible for facial harmony and aesthetics [1].

Hidalgo was the first to utilize a free composite fibular flap in mandible reconstruction and popularized it [2]. At present, the use of this flap is one of the most preferred reconstructive options in complex mandibular defects.

The free vascularized fibular flap, based on the peroneal artery and its commitant veins, can be transferred as purely osseous one or a combined osteocutaneous, myoosseous or osteomyocutaneous one. The main advantage of the fibular flaps is that they present with a constant topographical and microvascular anatomy, providing foreseen and reproducible operative results. Another major advantage is the opportunity for single stage immediate reconstruction of the resection defect, altogether with a minimal long term donor site morbidity, providing alongside that an adequate volume and quality of the reconstructed bone for further oral cavity dental implant rehabilitation.

The three dimensional (3D) virtual surgical planning (VSP) has gained popularity both in planning the resection volume and in the reconstructive aspects of head and neck surgery. It allows for detailed volume visualization of the operation zone before and after removing the pathologic segments and helps in precise reproduction of the virtual planning during the real operation by production of surgical guides. It also significantly reduces the time for segmentation and fixation of the fibular bone to the mandible site, which on its behalf reduces the flap's ischemic time, facilitates the precise donor/recipient bone contact and restores the mandibular bone anatomy and function.

There are two main ways of setting up virtual surgical planning, modelling and 3D implant printing in the clinical hospital practice - the use of an in-hospital 3D lab with relevant equipment and medical-engineering capabilities, or alternatively - an outsourcing company with appropriate portfolio. Establishing either of these ways is a strongly financially dependent undertaking, leading to very disproportionate development of the virtual surgical skills and methods within the different countries across Europe.

\section{MATERIALS AND METHODS:}

In order to introduce into clinical practice the entire process of virtual 3D planning, modelling and patient specific implant printing in fibula free flap mandibular reconstructions within Bulgaria's hospital practice, a medicoengineering collaboration with the Gebrüder Martin GmbH \& Co division (Tutlingen, Germany) of KLS Martin Group was established. 
By means of a thin-slice $(0.3-0.6 \mathrm{~mm})$ pre-operative computer tomography (CT) of the head and neck and a CT angiographic examination of the lower legs, both uploaded onto the IPS Gate ${ }^{\circledR}$ Server (KLS Martin Group), a virtual $3 \mathrm{D}$ resection/reconstruction modeling (fig. 1, 5) and a volume planning (fig. 3) was performed by dedicated web conferences, during which the technical parts of the operation procedures were simulated in details.

Post determination and confirmation of the resection lines (fig. 1), 3D printed surgical guides from autoclavable polyamide were constructed (fig. 2) and produced to aid in the precise removal of the pathological bone sections of the mandible. These guides were used during the actual surgical operations themselves.

Another set of guides - for segmentation of the fibular bone and its fixation to the mandible area were printed by the same material (fig. 4). Mandibular and fibular models were also produced to physically depict and verify the virtual planning, together with individual patient specific titanium implants, which were designed (fig. 5) and produced using a laser sintering method, to permanently stabilize the segmented fibula in a position of new mandibular bone at the end of the operations in each case (fig. 7, 8, 9),

Fig. 1. 3D Mandible disease presentation
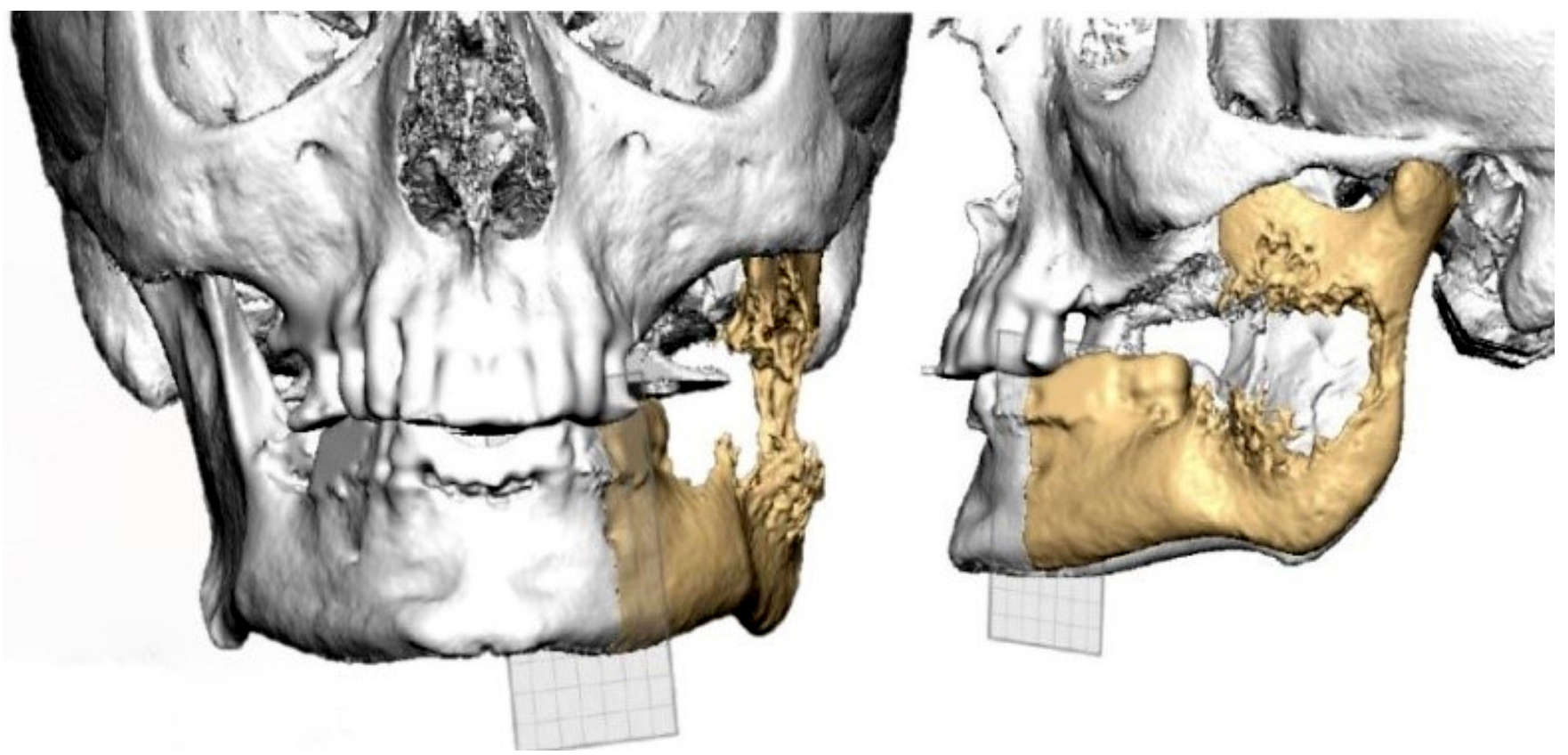

Fig. 2. Virtual mandible resection and surgical guide construction
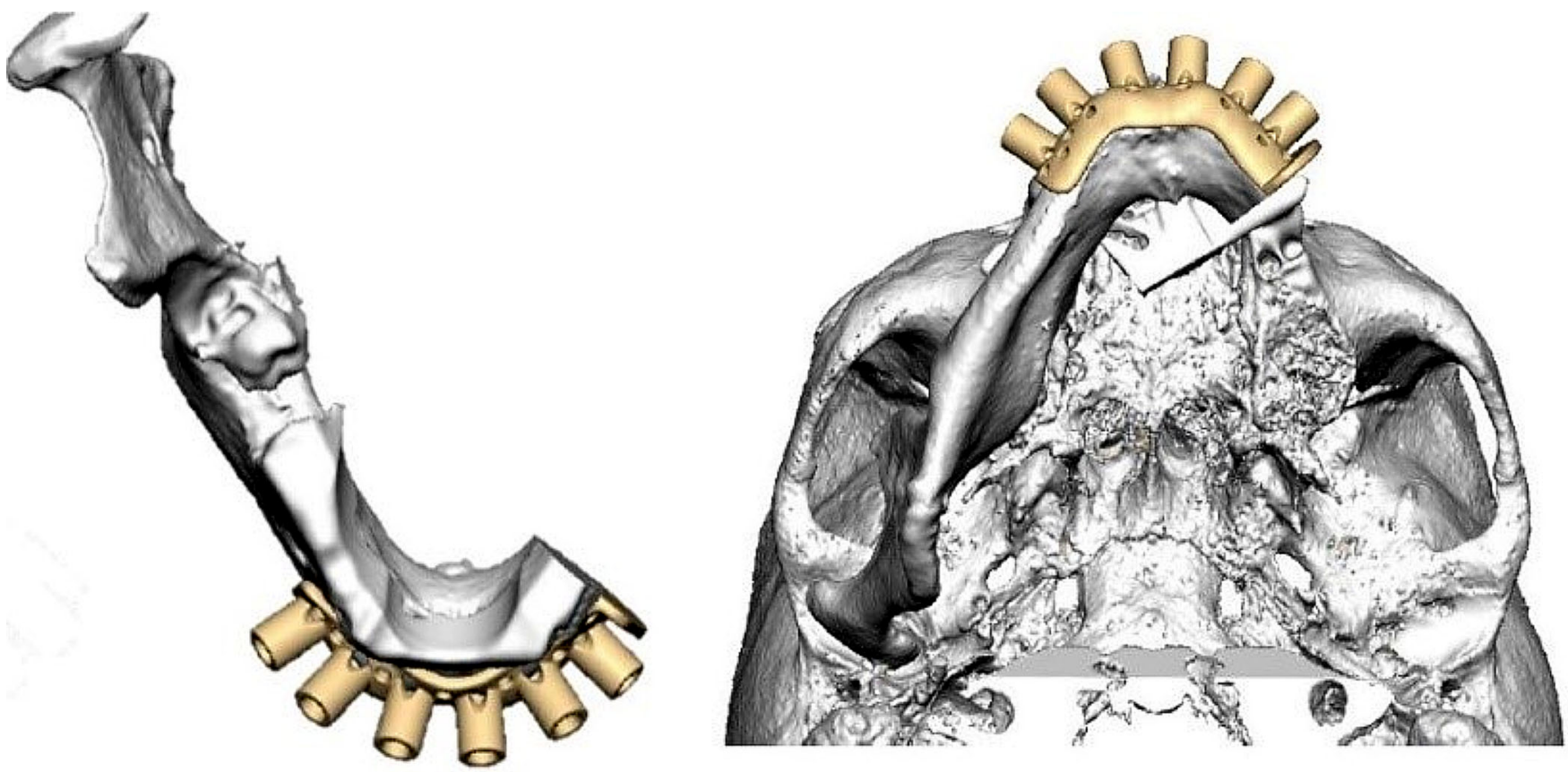
Fig. 3. 3D depiction of the left fibula donor site

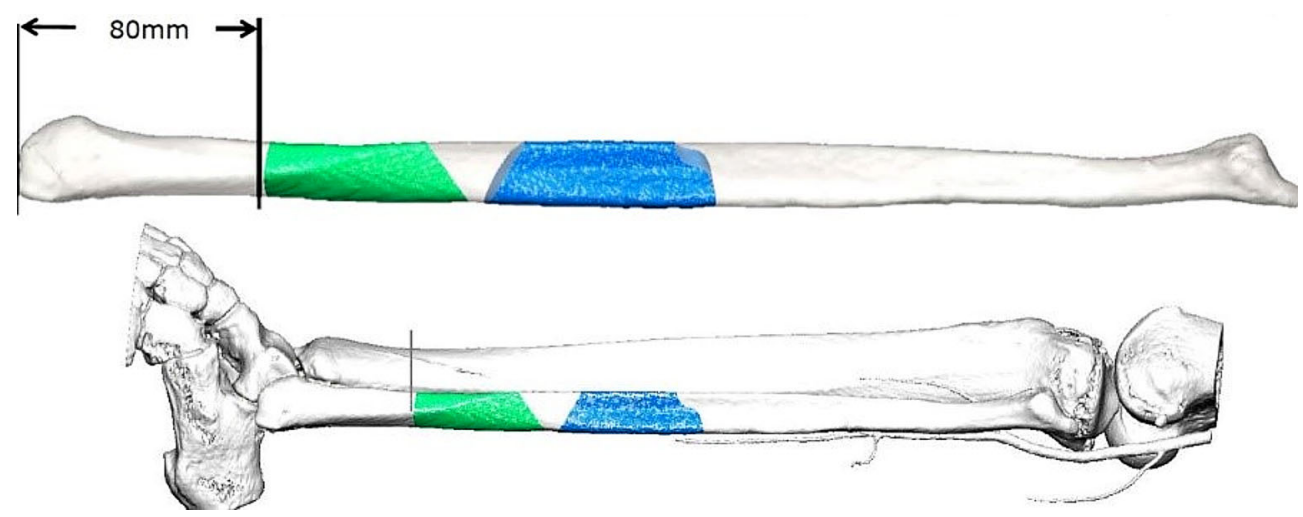

Fig. 4. Donor site surgical guides
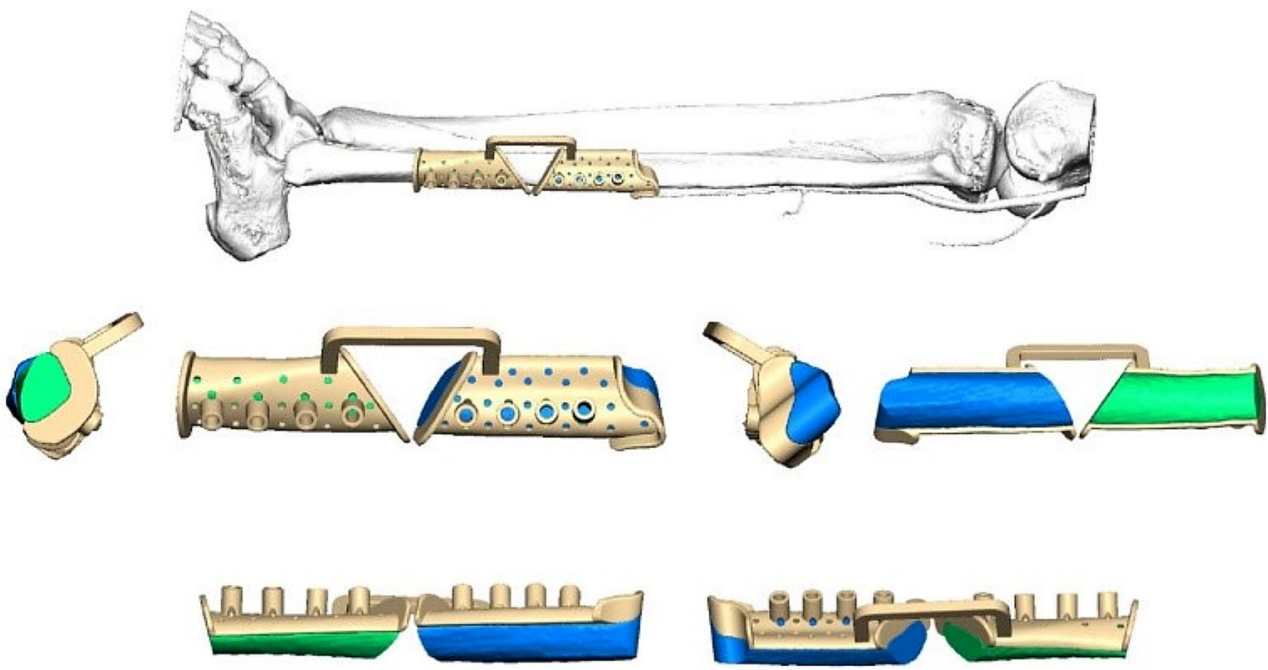

Fig. 5. Mandible defect fibula reconstruction and patient specific implant construction
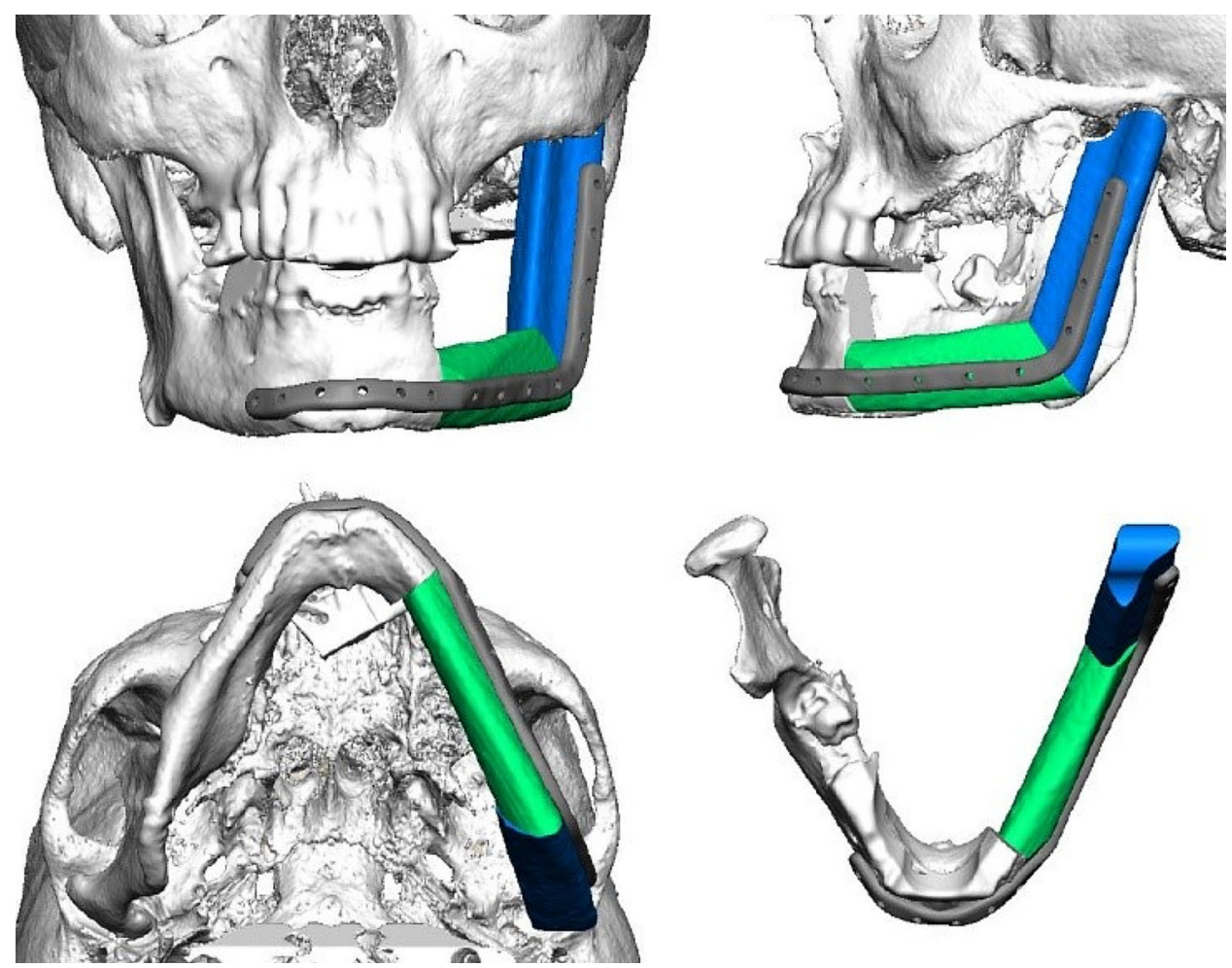
The length and width of the fixation screws in the area of the residual mandible and the fibular bone were determined during the process of virtual planning in the precision range of $0.1 \mathrm{~mm}$. In the final summary, the recom- mended summarized dimensions followed the standard equipment kit of the KLS Martin osteosynthesis screw set, which offered à $1 \mathrm{~mm}$ (for the length) and $0.3 \mathrm{~mm}$ (for the width) steps, respectively (fig. 6).

Fig. 6. Screw distribution for fixing the individual titanium implant to the mandible and the bone transplant.

The dimensions of the screws and the recommended lengths are indicated.

screws

\begin{tabular}{|l|c|c|c||}
\hline screw typ & pos. & $\begin{array}{c}\text { Measured } \\
\text { screw } \\
\text { length* }\end{array}$ & $\begin{array}{c}\text { Recommende } \\
\text { d screw } \\
\text { length }\end{array}$ \\
\hline $2,0 / 2,3$ Locking (bicort.) & 1 & 12,0 & 13 \\
\hline $2,0 / 2,3$ Locking (bicort.) & 2 & 11,7 & 13 \\
\hline $2,0 / 2,3$ Locking (bicort.) & 3 & 12,7 & 15 \\
\hline $2,0 / 2,3$ Locking (bicort.) & 4 & 13,0 & 15 \\
\hline $2,0 / 2,3$ Locking (bicort.) & 5 & 11,6 & 13 \\
\hline $2,0 / 2,3$ Locking (bicort.) & 6 & 12,4 & 13 \\
\hline $2,0 / 2,3$ Locking (monocort.) & 7 & & $7-9$ \\
\hline $2,0 / 2,3$ Locking (monocort.) & 8 & & $7-9$ \\
\hline $2,0 / 2,3$ Locking (monocort.) & 9 & & $7-9$ \\
\hline $2,0 / 2,3$ Locking (monocort.) & 10 & & $7-9$ \\
\hline $2,0 / 2,3$ Locking (monocort.) & 11 & & $7-9$ \\
\hline $2,0 / 2,3$ Locking (monocort.) & 12 & & $7-9$ \\
\hline $2,0 / 2,3$ Locking (monocort.) & 13 & & $7-9$ \\
\hline $2,0 / 2,3$ Locking (monocort.) & 14 & & $7-9$ \\
\hline \hline
\end{tabular}

"Measured screw length $=$ Plate thickness combined with bone thickness

\section{RESULTS:}

Three consecutive patients were operated on at the Specialized University Hospital for Maxillofacial Surgery and the University Hospital N.I.Pirogov, Sofia, between August-October 2017, using the virtual planning, modeling

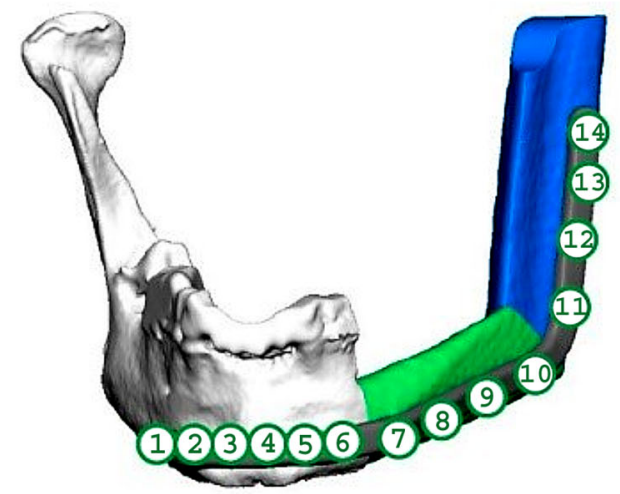

The screw threads are both for Locking and for Non-Locking (2.0/2.3 $\mathrm{mm})$
Table 1. Patients, histology and flap types

\begin{tabular}{|c|c|c|c|c|}
\hline Patient No. & Age & Gender & Diagnosis & Flap type \\
\hline MD & 43 & male & fibrosarcoma & osteomyocutaneous \\
\hline DG & 37 & male & ameloblastoma & osteal \\
\hline PP & 63 & male & chondrosarcoma & osteomyocutaneous \\
\hline
\end{tabular}

Mandible with or without lower facial third soft tistions were performed in each of the individual cases, assisted by resection guides, fibula segmentation guides and patient specific titanium sintered implants. (fig. 7, 8, 9). The expenses of the entire medico-engineering process were self-covered.

The case summaries are as follows:

\section{Patient 1}

MD, a 43 years old male, was diagnosed with a highly differentiated fibrosarcoma invading the left posterior mandible and the pterygomandibular, parotideomasseteric and buccal spaces. sue resections and single-stage microvascular reconstruc- and 3D printing methods described above. The initial biopsy investigations reported malignant mesenchymal tumors - fibrosarcoma and chondrosarcoma in two of the cases and a benign odontogenic tumor - ameloblastoma in the third one (table 1).
Post tracheostomy, a resection of the soft tissue component was performed in-block with the left mandible, distal to the first premolar and including the left condyle, with the help of the virtually prepared and 3D printed resection cutting guide. At the same time, a second team utilized the surgical reconstruction cutting guides to osteotomize and shape the left fibula bone while still connected to the donor site blood vessels. The microvascular revascularization of the flap at the recipient site was provided by micro-anastomosis of arteria peronea sinistra to arteria ligualis sinistra (end-to-end) and vena peronea sinistra to vena jugularis interna sinistra (end-to-side). 
Fig. 7. Patient 1 - mandibular and fibular models, surgical guides and patient specific implant
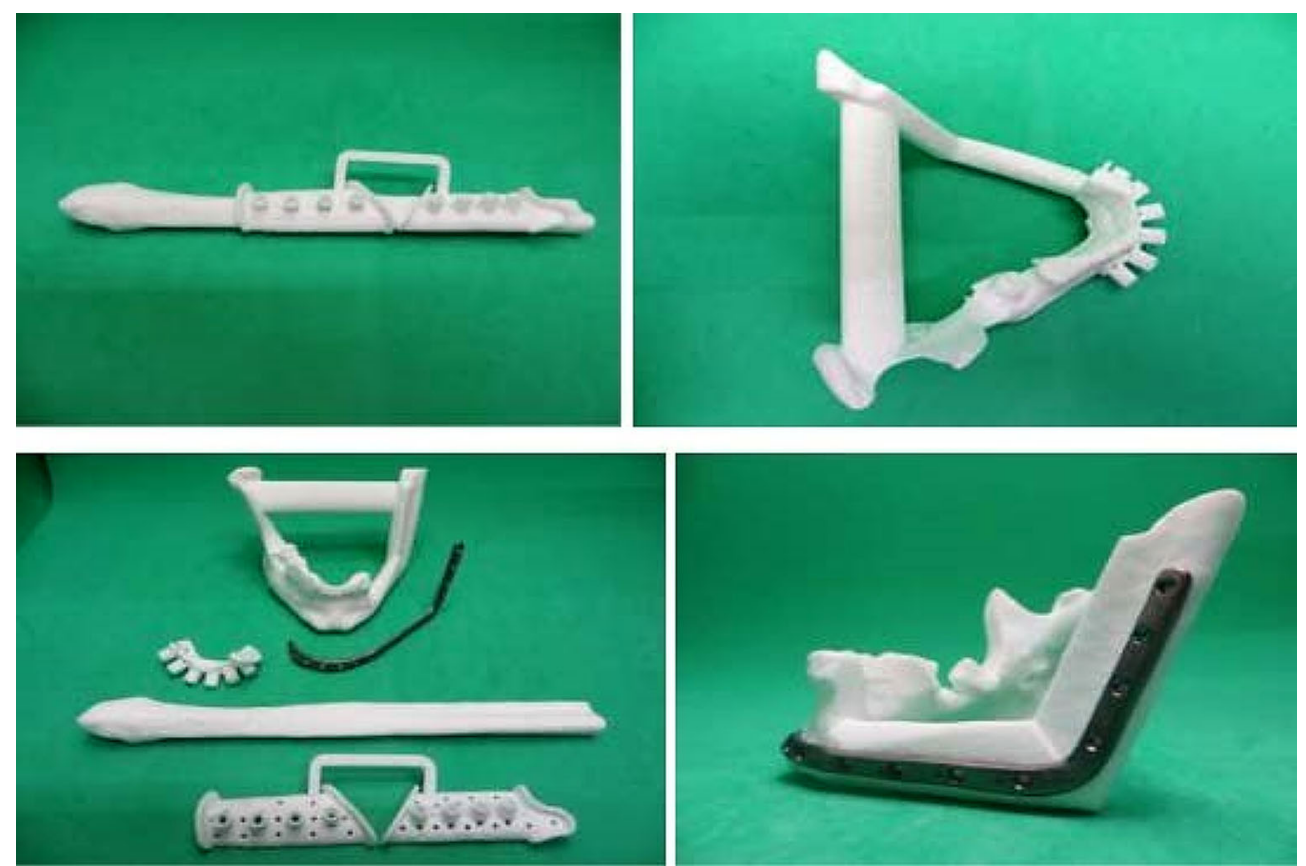

\section{Patient 2}

DD, a 37 years old male, was diagnosed with ameloblastoma of the right mandible body, angle and ramus.

Through a unilateral neck incision in a skin crease, a soft tissue dissection was performed together with preparation of the facial artery and vein for microvascular anastomoses. The basal rim was reached, and the mandible was exposed together with the expansive tumor process. With the help of surgical cutting guides, the resection lines were marked, and an osteotomy was performed.

At the same time, a second surgical team harvested a vascularized fibular bone flap from the right leg by reconstructive cutting guides. The transplant was transferred to the defect, and the microsurgical anastomoses were accomplished - arteria peronea dextra to arteria facialis dextra (end-to-end) and vena peronea dextra to $\mathrm{v}$. jugularis externa dextra (end-to-end).

Fig. 8. Patient 2 - mandibular and fibular models, surgical guides and patient specific implant

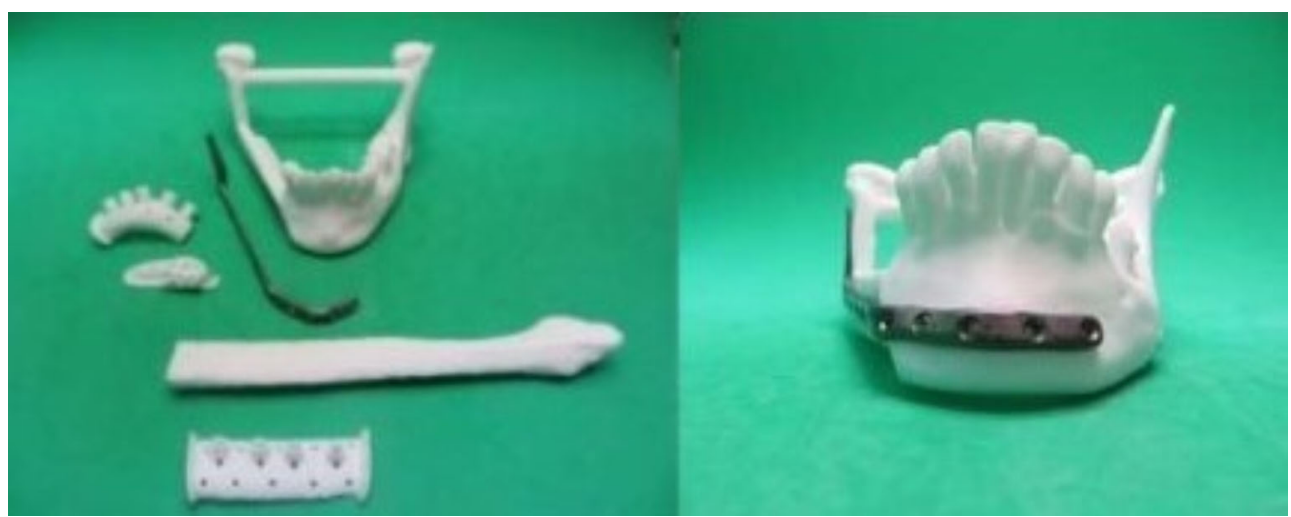

\section{Patient 3}

PP, a 62 years old male, was diagnosed with a long standing mandibular chondrosarcoma, extending to the whole of the submental, submandibular, mental, labial and buccal areas.

The operation involved tracheostomy, radical resection of the lower facial third soft tissues, in-block with a subtotal mandible resection by surgical cutting guides, sparing only the left mandibular angle and ramus. A bilateral selective level I-III neck dissection was also performed together with exposure and preparation of the recipient neck blood vessels. At the same operative time, a composite vascularized 4 segments osteomyocutaneous fibular flap from the left lower leg was formed by the reconstructive team. The fibula was segmented while still vascularized in the leg by reconstructive cutting guides and was fixed to the preoperatively produced patient-specific reconstructive mandible implant. The flap was then transferred to the head and neck area and microsurgically anastomosed - arteria facialis sinistra to a. peronea sinistra (end-to-end); v.peronea sinistra 1 to $\mathrm{v}$. facialis sinistra (end-to-end) and v. peronea sinistra 2 to $\mathrm{v}$. thyreoidea sinistra (end-to-end). 
Fig. 9. Patient 3 - mandibular and fibular models, surgical guides and patient specific implant
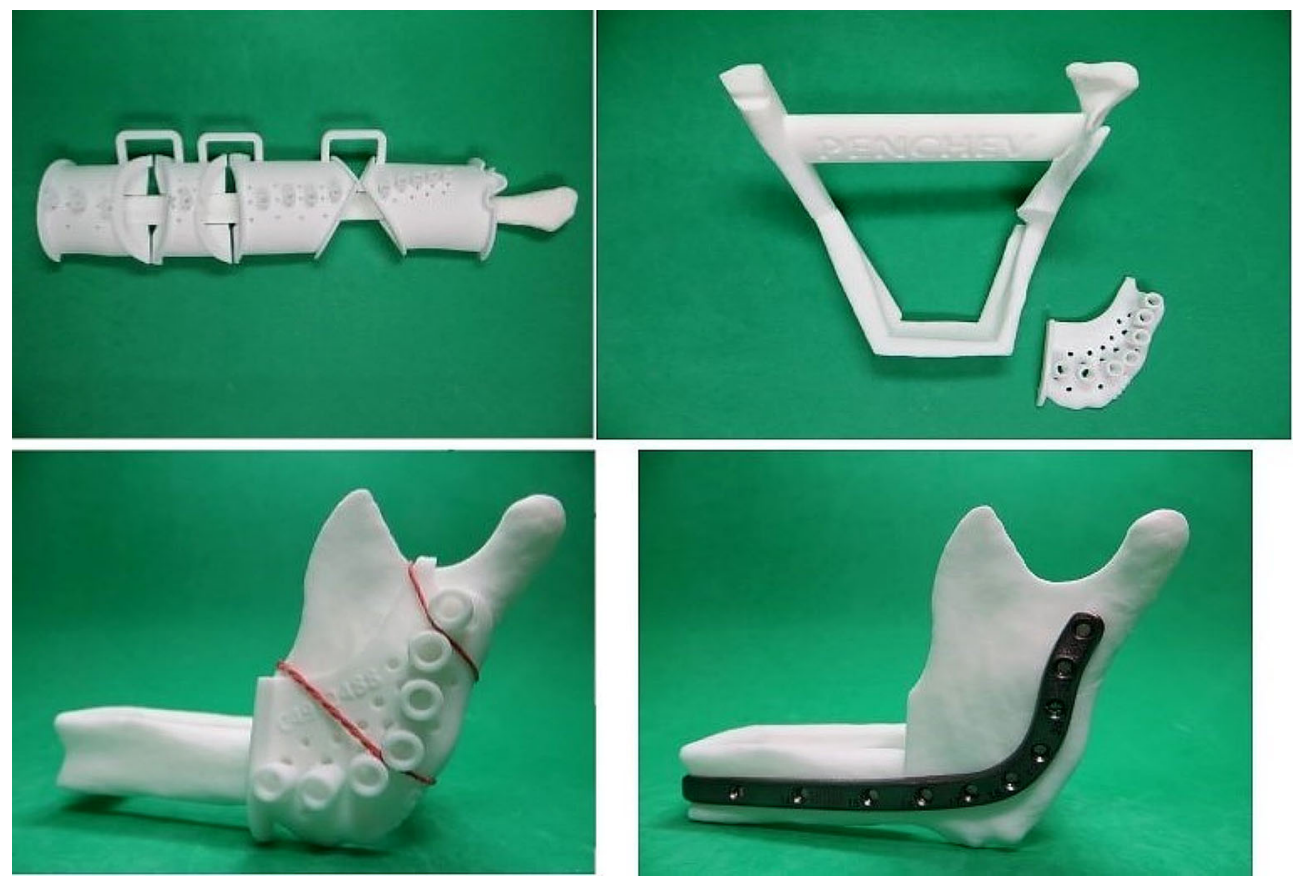

\section{DISCUSSION:}

The initial reports regarding virtual surgical planning (VSP) go back to the early 1990s. Rose et al. described a series of six patients in whom computer-generated models based on CT data were used for selection of the appropriate donor sites [3]. In 2005, Eckardt and Swennen were the first to make a virtual plan of the desired mandible shape, using virtual voxel-based planning [4]. A metal template was created based on the virtual geometric data to facilitate optimal contouring of the fibular bone graft.

Although the usefulness of this application was immediately recognized, it was utilized in clinical practice no earlier than several years later due to the complex, time consuming, and expensive nature of the method.

Thankappan et al. described a VSP process in which the healthy bone side was mirrored over the affected one [5]. In the next year, Hirsch et al. introduced a VSP concept that combined planning and patient-specific stereolithographic cutting guides for mandibular resection and fibula segmentation [6].

These continuous efforts aimed at facilitating and upgrading the standardized treatment method of choice in extensive head and neck resection defects - the comprehensive microvascular reconstruction. [7, 8, 9].

The surgical interventions for removal of complex head and neck tumors with immediate microvascular defect reconstruction usually have a duration in the wide range of 8-16 hours. At the same time, the jaws have a unique three-dimensional form and have no analogue in the human body. In the absence of precise pre-operative planning and modeling, each of the individual reconstructive aspects, especially the bone fragments length and form, should be measured and re-established intraoperatively, resulting in significant operative time delay. And while the length of the mentum, the body, or the ramus of the mandible could be relatively accurately measured by a plain surgical ruler, the three-dimensional reconstruction of the symmetry (in particular the transverse and rotational position of the segments) of the mandible, the position of the articulating mandibular processes, as well as the optimal contact between the individual fibular segments or between the fibula and the mandibular bone are extremely difficult for intraoperative accomplishing.

Another very important positive feature of virtual planning, combined with the production of reconstructive cutting guides, is the reduction of ischemic time for bone graft processing and transfer, which in its turn is vital for the success of tissue revascularization.

Digital planning and patient specific implant production technologies have upraised the head and neck reconstructive surgery to a next level, which unfortunately is not evenly accessible across Europe or the rest of the world, due to the very different nature of health systems regulations. The Bulgarian National Insurance Fund (NHIF), for example, which is the sole public legal entity that carries out and administers the compulsory health insurance in Bulgaria and in-practice regulates all the major public hospital treatment of Bulgarian citizens, does not finance the implementation of any implants or devices for maxillofacial / head and neck oncology, deformity, trauma or reconstruction surgery. This fact significantly hampers the widespread implementation of the contemporary facial bone surgery approaches and leaves them dependent on either patient self-funding or other types of private funds, which on their behalf are quite sporadic.

On the other hand, the use of contemporary digital surgical and engineering technology is so prominently beneficial that sooner rather than later, a constant recognition by the different health regulating authorities is inevitable for the benefit of patients and surgical practice development. 


\section{CONCLUSION:}

The first case series of virtual planning, modeling and patient specific implant printing in fibula microvascular free flap reconstructions of mandibular defects, introduced an important surgical concept to Bulgaria's health service system and demonstrated the precision, reproducibility and accuracy of this contemporary surgical approach.

\section{ACKNOWLEDGEMENT}

The authors would like to express their sincere gratitude to the management team of the "Pirogov" University
Hospital in Sofia, and in particular to its Director - Prof. Dr. Assen Baltov, PhD and its Vice-director and Head of the Neurosurgery Department - Prof. Dr. Nikolay Gabrovsky, $\mathrm{PhD}$, for their exceptional cooperation and support in the realization of the 3D digitally assisted head and neck surgery in Bulgaria.

We would also like to express our deep gratefulness to the Manager of the Specialized University Hospital for Maxillofacial Surgery, Sofia, Dr. Genadi Genadiev and to the entire hospital team, who sincerely supported the introduction and development of the regular microsurgical free flap reconstructive head and neck service.

\section{REFERENCES:}

1. Becker ST, Menzebach M, Küchler T, Hertrampf K, Wenz HJ, Wiltfang J. Quality of life in oral cancer patients-effects of mandible resection and socio-cultural aspects. $J$ Craniomaxillofac Surg. 2012 Jan;40(1):24-7. [PubMed]

2. Hidalgo DA. Fibula free flap: a new method of mandible reconstruction. Plast Reconstr Surg. 1989 Jul;84(1):71-9. [PubMed]

3. Rose EH, Norris MS, Rosen JM. Application of high-tech three-dimensional imaging and computer-generated models in complex facial reconstructions with vascularized bone grafts. PlastReconstr Surg. 1993 Feb;91(2):252-64. [PubMed]

4. Eckardt A, Swennen GR. Virtual planning of composite mandibular re- construction with free fibula bone graft. J Craniofac Surg. 2005 Nov; 16(6):1137-40. [PubMed]

5. Thankappan K, Trivedi NP, Subash P, Pullara SK, Peter S, Kuriakose MA, et al. Three-dimensional computed tomography-based contouring of a free fibula bone graft for mandibular reconstruction. J Oral Maxillofac Surg. 2008 Oct;66(10): 2185-92. [PubMed]

6. Hirsch DL, Garfein ES, Christensen AM, Weimer KA, Saddeh PB, Levine JP. Use of computer-aided design and computer-aided manufacturing to produce orthognathically ideal surgical outcomes: a paradigm shift in head and neck reconstruction. J Oral Maxillofac Surg. 2009 Oct; 67(10):2115-22. [PubMed]
7. Ragbir M, Brown JS, Mehanna H. Reconstructive considerations in head and neck surgical oncology: United Kingdom National Multidisciplinary Guidelines. J Laryngol Otol. 2016 May;130(S2): S191-S197. [PubMed]

8. Cannady SB, Lamarre E, Wax MK. Microvascular Reconstruction: Evidence-Based Procedures. Facial PlastSurgClin North Am. 2015 Aug; 23(3): 347-56. [PubMed]

9. Markiewicz MR, Bell RB, Bui TG, Dierks EJ, Ruiz R, Gelesko S, et al. Survival of microvascular free flaps in mandibular reconstruction: A systematic review and meta-analysis. Microsurgery. 2015 Oct;35(7):576-87. [PubMed]

Please cite this article as: Yanev N, Slavkov S, Markov D, Malinova A. Three dimensional virtual planning, modelling and printing in fibula free flap mandibular reconstructions - initial case series in a limited resource environment. $J$ of IMAB. 2021 Apr-Jun;27(2):3752-3758. DOI: https://doi.org/10.5272/jimab.2021272.3752

Received: 27/07/2020; Published online: 31/05/2021

Address for correspondence:

Nikolay Yanev, DMD, MD, PhD, FEBOMFS

Maxillofacial Surgery Unit, University Hospital N.I.Pirogov

21, Gen.Totleben Str., Sofia 1606, Bulgaria.

E-mail: nyanev@abv.bg 